\title{
Malpresentations and Malpositions
}

\section{Background}

1. Definition

- Presentation:

- Fetal part presenting at pelvic outlet

- Normal:

- Vertex head is presenting part

- Malpresentations

- Cephalic

- Brow (brow of infant is presenting), face and compound (head and hand presenting together)

- Breech (complete, frank, footling)

- Shoulder

- Position: position of fetal occiput in relation to maternal pelvis

- Normal: occiput anterior

- Malpositions: occiput posterior, occiput transverse

\section{Pathophysiology}

1. Incidence

- Face $1 / 600$

- Brow $1 / 1,400$

- Breech $1 / 33$

- Compound 1/1,500

- Occiput posterior: $15 \%$ early labor and $5 \%$ at delivery

- Transverse $1 / 335$

2. Risk factors for malpresentation

- Fetal factors

- Prematurity

- Large baby

- Fetal anomaly:

- Anencephaly, hydrocephaly, muscle dystrophy, neurologic impairment, anterior neck mass or multiple nuchal cord loops (prevent flexion of the head); $50 \%$ of face presentation

- Short umbilical cord

- Fetal death

- Maternal and uterine factors for malpresentation

- Contracted pelvis e.g. distortions following pelvic fracture that prohibits fetal descent into the pelvis

- Multiparity resulting in lax abdominal wall

- Multiple pregnancy

- Uterine cavity abnormalities (bicornuate, septate uterus)

- Space occupying lesions (uterine leiomyomatoma)

- Placental abnormalities (placenta previa)

- Amniotic fluid volume abnormalities (oligo-polyhydramnios)

3. Morbidity/mortality

- Increased likelihood of intervention due to anomalies on fetal tracing 
- Lower baseline often seen with occiput posterior position, ineffective uterine action, feto-pelvic disproportion, prolonged or arrest of labor

- Increased PROM increasing risk of infection and cord prolapse

- Pathologic contraction rings

- Usually the result of unrecognized labor dystocia and possible rupture of lower uterine segment

- Increased incidence of instrumental delivery or C-section (maternal trauma, bleeding, infection)

\section{Diagnostics}

1. History

- No descent of baby late in pregnancy in many cases of malpresentation

- Fetal head in the fundas for breech and elsewhere for other malpositions

- Large abdomen suggestive of failure of fetus to descend

- Fundal height measurement may confirm on physical exam

2. Physical exam

- Leopold maneuvers

- Vaginal exam

- Head unengaged after rupture of membranes

- Face

- Triangle shaped: mouth and orbital ridges (or malar bones)

- Chin is the presenting part, anteriorly or posteriorly

- Brow:

- Frontal sutures, anterior fontanelle, orbital ridges, eyes but not mouth nor chin

- Breech

- Great trochanters (or ischial tuberosities) + anus in a line (attn: mouth can be mistaken for anus and ischial tuberosities for malar bones)

- Compound

- Fetal extremity prolapses along with presenting part

- Transverse lie

- Ribs, scapula, clavicle, axilla

- Described as dorsoanterior, dorsoposterior and right or left acromial

- Prolapse of arm or hand in vagina

\section{Diagnostic testing}

- Based upon physical examination

- Ultrasound may be useful to confirm breech and transverse presentations

- Ultrasound not useful for OP/OA

\section{Management}

1. External cephalic version for breech/transverse lie with or without induction of labor after 37 weeks

2. Criteria for external version >37 weeks; singleton; unengaged presenting part; reactive NST; good amniotic fluid volume

3. Contraindication to external version 
- Previous unexplained 3rd trimester bleed; placenta previa; prior classical c/s; previous myomectomy; oligohydramnios; PROM; abnormal U/S; suspected IUGR; hypertension, signs of uterine placental insufficiency

- Success rate of version is operator dependent

- Up to $60-70 \%$

- Risk of non -reassuring fetal monitoring

- Done in settings where emergent delivery can be done

4. Augmentation of labor not contraindicated if no signs of obstruction and evidence of hypotonia, except in brow and compound presentations

5. Specifics:

- Face

- 25-30\% : spontaneous rotation of mentum posterior to mentum anterior

- Mentum anterior can deliver vaginally while mentum posterior cannot

- Neonatal resuscitation endotracheal intubation may be difficult secondary to facial swelling

- Instrumentation usually contraindicated, vacuum extractor contraindicated

- C-section most common form of delivery

- Brow

- Spontaneous conversion to face $(30 \%)$ or occiput posterior $(20 \%)$

- C-section most common form of delivery

- Breech

- Planned c-section for persistent breech

- Term Breech Trial : planned c-section for term breech associated with decrease perinatal mortality and morbidity; modest increase in shortterm maternal morbidity

- Criteria for vaginal breech delivery:

- $\quad>36$ weeks; adequate clinical pelvimetry; complete or frank breech; fetus not too large; fetal head flexed; no other indication for c/s; no previous c/s for CPD; absence of fetal anomaly that may interfere with vaginal delivery; continuous fetal heart rate monitoring available; experienced physician,

- Informed consent with women planning a vaginal breech delivery should include the risk of neonatal morbidity and mortality may be higher than if a planned c-section is performed

- Compound

- In most of the cases, prolapsed part will ascend with the descent of the presenting part, allowing normal delivery

- Most usual presenting part is the hand or arm

- Instrumentation not recommended

- Transverse lie/shoulder

- Risk of formation of pathologic contraction ring above lower uterine segment and uterine rupture if shoulder forced in the pelvis

- Delivery by Caesarean section if no version to vertex

- Occiput posterior

- $90 \%$ conversion to occiput anterior during labor

- Spontaneous vaginal delivery in OP position possible

- Vacuum or forceps may be needed 
- Manual rotation of persistent OP may assist in facilitating birth

- Occiput Transverse

- If does not convert to occiput anterior or occiput posterior will need either forceps rotation or Caesarean

See also Vacuum-assisted delivery

\section{References}

1. Gabbe: Obstetrics - Normal and Problem Pregnancies, 4th ed., 2002, Churchill

Livingstone, Inc., pp. 473-498

2. Williams Obstetrics, 22nd ed., 2005, McGraw-Hill Companies, Inc., pp.506-513

3. Uptodate: www.uptodate.com

4. World Health Organization: www.who.int

5. SOGC clinical guidelines

6. ALSO manual

Authors: Lisa Graves, MD, \& Delphine Laramee, MD, Trillium Health Care, ON

Editor: David Wakulchik, MD, Aultman FMRP, $O H$ 